\title{
Sound Absorptions by Micro-Perforated Panels
}

Noise is omnipresent! Once generated, it is difficult to be dealt with. Industries are increasingly aware of the growing importance of vibration and noise problems, which calls for their due consideration at the early design stage for any manufactured products. Industry wishes to distance itself from the approach that relies on after-the-fact palliatives. Meanwhile, technical progress does not necessarily go hand in hand with a decrease in the noise level. The desire of building lighter goods with increased performance is usually accompanied by a degradation in the acoustic quality. These compounding factors make noise abatement a challenging task, calling for innovative engineering solutions and noise mitigation methods (passive or active).

Sound absorptions are one of the important means for noise mitigation. Conventional sound absorption materials, such as porous and fibrous materials, are based on thermal and viscous losses inside the pores and fibres. Apart from environmental concerns, they usually need a bulky volume to achieve low frequency absorptions. In that regard, Micro-perforated panels (MPPs) show advantages. A MPP is a thin sheet with a lattice of sub-millimetre size perforations distributed over its surface. The perforations, at such a scale, provide considerable acoustic resistance and low acoustic reactance, conducive to sound absorption without the use of any porous material. A conventional MPP absorber takes the form of a MPP fitted in front of a backing wall with an air gap between them. The depthdependant stiffness of the backing cavity and the mass inside the hole generate resonance-type absorptions. Its design procedure was proposed by Chinese scholar Maa in the 1970s, which become the cornerstone for the subsequent research on MPPs.

Early applications of MPPs were mainly in architectural acoustics and environmental noise control, where simple acoustic fields are usually considered. In these applications, a MPP is usually treated as a locally reacting sound absorption material with its surface impedance or sound absorption coefficient measured either in a reverberation chamber or a Kundt tube. Later on, MPPs were used in more complex and compact mechanical systems, exemplified by MRI scanners, cooling systems, nacelles of turbofan engines and interiors of engine enclosures etc. The common feature of these applications is that a MPP may closely interact with the surrounding vibroacoustic elements. This calls for revisiting and interrogating the in-situ sound absorption mechanism of the MPP. Sure enough, the working principle and the sound absorption efficiency of the MPPs are found to greatly depend on the surrounding vibroacoustic environment. Demonstration was made using a simple MPP with a cavity backing, subject to oblique and diffuse sound incidence. Whilst posing challenges to the modelling and analyses, the observed phenomena also offer opportunities to cater for particular applications. For example, the shape of the backing cavity, as well as their modal behaviour, can be tuned to customize the effective sound absorption range of an absorber. Another example is MPPs in enclosures. Although different backing arrangements may provide seemingly similar sound absorption curves from the standard impedance tube measurement, the in-situ sound absorptions can be drastically different. Results point at the necessity of treating a MPP structure as part of the entire system, rather than an absorption boundary characterized in simple acoustic environment.

The presence of flow poses great challenges in the characterization of MPPs. CFD simulations confirm the sound absorption mechanism of the MPP absorbers in different grazing flow regimes and sound pressure levels (SPL). At low SPL, viscous effect near the hole dominates the energy dissipation; while at high SPL, sound energy is mainly converted into the kinetic energy to generate vortex shedding and energy dissipation. In the moderate SPL range, viscous effect, resistant effects of the vortex in the orifice, movement of the shear layer and vortex shedding would all contribute. The dominant level of each, however, depends on factors such as the orifice size, SPL and flow speed. Attempts have been made to develop new formulae for the prediction of acoustic impedance of MPPs with grazing flow. The latest one is the model based on the velocity gradient in the viscous sublayer over the panel surface, in which a flow-related term was added to Maa's impedance formula. The accuracy of the model was compared with existing ones reported in the open literature, showing encouraging performance in terms of both prediction accuracy and application range. The formulae were used to investigate MPP liners in flow ducts, thus providing guidelines to the design and optimization of such devices for flow-duct noise abatement.

Up to now, various academic cases and practical design, analysis and optimization problems have been reported. Typical application examples include ducted ventilation system and windows, noise barriers, MPP housing devices, silencers, mufflers and domestic products etc. Taking a duct liner as an example, studies reveal a hybrid noise attenuation mechanism of the MPPs. As a result, the hole diameter, together with the perforation ratio and other parameters, can be optimized to balance the dissipative and reactive effects, for ultimately achieving the desired broadband noise attenuation within a prescribed frequency region.

The concept of MPP for sound absorption is intuitive and easy to understand. However, industrial people are sometimes puzzled by their bitter observation that its implementation in their product does not always deliver the expected results, although impedance tube testing on sound absorption tells otherwise. Therefore, the "how" and "why" part of the problem is vital, not only for academia but also for practitioners. Although MPP based technologies have found their widespread uses in conventional areas, new impetus and ideas are still needed. Encouraging progress includes acoustic metamaterials using MPP-based periodic lattice and small scale subwavelength sound absorbers for low frequency sound absorptions. These new attempts would offer new possibility and open new horizons for more innovative utilizations of MPPs which go beyond their traditional design and applications.

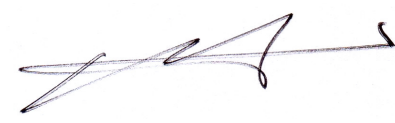

\section{Li Cheng}

IIAV board director 